\title{
Bio-inspired clay nanosheets/polymer matrix/mineral nanofibers ternary composite films with optimal balance of strength and toughness
}

\author{
Shi-Kuo Li, Li-Bo Mao, Huai-Ling Gao, Hong-Bin Yao and Shu-Hong Yu*
}

\begin{abstract}
Although remarkable progress has been witnessed in mimicking the nacre-like architecture in laboratory, it remains a great challenge for understanding the unique balancing mechanism of toughness and strength in biological materials. Here, taking advantage of the synergistic effect of different dimensional nanoscale building blocks, we fabricate nacre-like films that reconcile high strength and toughness. The obtained ternary lamellar composite films are constructed by one-dimensional xonotlite nanowires and two-dimensional montmorillonite nanosheets with the assistance of poly(vinyl alcohol). The ternary composite films show high strength ( $(241.8 \pm 10.2) \mathrm{MPa})$ and toughness $\left((5.85 \pm 0.46) \mathrm{MJ} \mathrm{m}^{-3}\right)$, both of which are higher than that of the single nanofibrillar xonotlite network films or the binary montmorillonite/poly(vinyl alcohol) composite films. The excellent mechanical properties of the nacre-like ternary composite films are aroused by the synergistic toughening mechanism of the different dimensional building blocks. This strategy provides a facile approach to integrate the nacre-like composite films with potential applications in tissue engineering scaffold, strong air barrier coatings, and fire-retardant packing materials.
\end{abstract}

Keywords: artificial nacre, mineral nanofibers, ternary lamellar, mechanical properties, vacuum filtration assembly

\section{INTRODUCTION}

Layered nacre provides a well-recognized structural design model for combining high stiffness and strength with remarkable toughness via the elegant hierarchical structures [1-4]. The excellent mechanical properties originate from the ordered layered arrangement of "brick-andmortar" architectures with well-tailed interface, which results in a 3000-fold improvement in toughness compared to aragonite monolith [5-8]. Mesocrystalline aragonite platelets (95 vol.\%) act as bricks for reinforcing and load bearing, and the rest biopolymers (5 vol.\%) are the soft mortar for inelastic shearing, viscoelastic deformation, frictional sliding and molecular energy-dissipating [9-11]. The organic phase mainly consisting of chitin nanofibrils, silk fibroins and soluble proteins guides the aragonite platelet growth through a biomineralization process [12-15]. Such an ordered "brick-andmortar" architecture with excellent mechanical properties has inspired enthusiasm to mimic the layered hard-soft arrangement by using different two-dimensional (2D) inorganic platelets and biopolymer matrices via layer-bylayer (LBL) assembly [16,17], spin coating [18,19], vacuum filtration assembly [20-23], air-water interface assembly [24], electrophoretic deposition [25], ice-template assembly [26], or evaporation-induced assembly [12,27]. Although many successful cases has been achieved in mimicking artificial nacre-like composites with excellent toughness and strength, most researches focus on stacking 2D platelets with polymers into a binary "brick-andmortar" architecture [28-30]. One-dimensional (1D) chitin nanofibrillar presented in the soft matrices, i.e., alternately stacking on the aragonite platelets in nacre [31-33], is often overlooked. Thus, to take full advantage of extraordinary mechanical properties of the natural nacre design, it is important to investigate ternary artificial "brick-and-mortar" architecture based on 1D and 2D building blocks [34-37].

Herein, we demonstrate that the strength and toughness of an artificial nacre-like architecture can be improved via the assembly of different dimensional building

Division of Nanomaterials \& Chemistry, Hefei National Laboratory for Physical Sciences at the Microscale, Collaborative Innovation Center of Suzhou Nano Science and Technology, Department of Chemistry, Chinese Academy of Sciences Center for Excellence in Nanoscience, Hefei Science Center of Chinese Academy of Sciences, University of Science and Technology of China, Hefei 230026, China

* Corresponding author (email: shyu@ustc.edu.cn) 
blocks, i.e., montmorillonite (MTM) nanosheets, and nanofibrillar xonotlite (NFX). 2D MTM nanosheets with high aspect ratios have been widely used to fabricate nacre mimics because of their hydrophilic surface, which adheres poly(vinyl alcohol) (PVA) molecules via hydrogen bonding and covalent bonding interactions $[8,17,38]$. $1 \mathrm{D}$ rigid NFX with tens of nanometers in diameter also has a sufficient surface area to adsorb PVA molecules by hydrogen bonding interactions. These building blocks can be well dispersed in distilled water and spontaneously stacked into the "brick-and-mortar" architecture via the vacuum filtration assembly. Both the ultimate tensile strength and toughness of the ternary composite films are much higher than that of the unitary NFX network films, the binary MTM/PVA composite films and the previously reported artificial clay/polymer binary composites. This study provides a simple method to design novel nacrelike composites with multi-dimensional building blocks based on the synergistic toughening mechanism.

\section{EXPERIMENTAL SECTION}

\author{
Materials \\ PVA $\left(M_{\mathrm{w}}=146,000-186,000\right.$, hydrolysis=99 wt.\% $)$ was \\ purchased from Sigma-Aldrich. $\mathrm{Ca}\left(\mathrm{NO}_{3}\right)_{2}, \mathrm{Na}_{2} \mathrm{SiO}_{3}$, and \\ anhydrous ethanol were obtained from Sinopharm Che- \\ mical Reagent Co., Ltd. $\mathrm{Na}^{+}$-type MTM was purchased \\ from Zhejiang Fenghong Clay Co. Ltd. without further \\ purification.
}

\section{Instruments}

Scanning electron microscopy (SEM) images were obtained with a field emission scanning electron microscope (S-4800, Hitachi) at an acceleration voltage of $5 \mathrm{kV}$. The acceleration voltage was increased to $10 \mathrm{kV}$ for the energy-dispersive X-ray (EDX) measurements. Transmission electron microscopy (TEM) was performed using a JEOL JEM-2100 instrument at $200 \mathrm{kV}$. X-ray diffraction (XRD) experiments were carried out with a Japan Rigaku Dmax $-\gamma$ A X-ray diffractometer with $\mathrm{Cu} \mathrm{Ka}$ radiation $(\lambda$ $=1.54178 \AA$ ). Thermal gravimetric analysis (TGA) was conducted on a TG/DTA 6300 (SII Nano technology Inc., Japan) thermal analyzer at a heating rate of $10^{\circ} \mathrm{C} \mathrm{min}^{-1}$ under air. Atomic force microscope (AFM) images were acquired using a Veeco Multimode VIII AFM. The mechanical properties were measured in the tensile mode in a universal mechanical testing machine (Instron 5565A). The tested rectangular strips were about $25 \mathrm{~mm}$ in length and $3 \mathrm{~mm}$ in width. The distance between the clamps was $10 \mathrm{~mm}$ and the load speed was $0.5 \mathrm{~mm} \mathrm{~min}^{-1}$. The cross- section width and thickness were carefully determined by SEM. Hardness performances were performed on a Nano Indenter G200 system (Agilent Technologies) based on a load-control method. Light transmittance spectra of the films were measured using a Hitachi U-4100 UV-vis spectrophotometer.

\section{Synthesis of xonotlite $\left[\mathrm{Ca}_{6}\left(\mathrm{Si}_{6} \mathrm{O}_{17}\right)(\mathrm{OH})_{2}\right]$ nanowires}

Xonotlite nanowires were synthesized according to a previous method with minor modification [39]. In a typical process, $200 \mathrm{~mL}$ of $0.5 \mathrm{~mol} \mathrm{~L}^{-1} \mathrm{Ca}\left(\mathrm{NO}_{3}\right)_{2}$ solution was dropwise added to $200 \mathrm{~mL}$ of $0.5 \mathrm{~mol} \mathrm{~L}^{-1} \mathrm{Na}_{2} \mathrm{SiO}_{3}$ solution at room temperature. After stirring for $1 \mathrm{~h}$, the white suspension was transferred into $500 \mathrm{~mL}$ Teflonlined stainless-steel autoclaves and kept at $200^{\circ} \mathrm{C}$ for $24 \mathrm{~h}$ with naturally cooling down to room temperature. Afterwards, the suspension was filtrated, and the solid was washed with distilled water and anhydrous ethanol alternatively for several times. Subsequently, the obtained nanowires were dispersed in distilled water to form a suspension (0.3 wt.\%) for further use.

\section{Vacuum filtration assembly of nacre-like MTM/PVA/NFX} ternary composite films

The suspension of MTM nanosheets was prepared as follows: a certain amount of MTM powers were dispersed in distilled water under stirring for one week. Then, the solution was centrifuged at $3000 \mathrm{rpm}$ for $10 \mathrm{~min}$ to remove the unexfoliated MTM. The transparent supernatant was collected, and the content of MTM was measured after a solution dried and re-weighed process. The mass concentration of the obtained MTM supernatant was about $0.3 \mathrm{wt} . \%$. PVA powder was dissolved in distilled water at $95^{\circ} \mathrm{C}$ under slow stirring to form an aqueous solution (10 wt.\%) before use. $10 \mathrm{~mL}$ of $10 \mathrm{wt} . \%$ PVA solution was gradually added to the above $150 \mathrm{~mL}$ of MTM dispersion solution under stirring for $12 \mathrm{~h}$ to allow maximize polymer adsorption. Subsequently, $100 \mathrm{~mL}$ of 0.3 wt.\% NFX solution was added into the above mixed solution dropwise and stirred vigorously for another $12 \mathrm{~h}$ to allow the adhesion of PVA onto the NFX surface. After that, the stabilized colloid solution was collected by centrifugation at $8,000 \mathrm{rpm}$ for $10 \mathrm{~min}$, washed by distilled water to remove the excess polymer. Finally, the obtained MTM/PVA/NFX ternary composite was re-dispersed into $40 \mathrm{~mL}$ distilled water under ultrasonication. The obtained homogeneous solution was vacuum filtered to form MTM/PVA/NFX ternary composite film on the cellulose acetate filtration paper (pore size, $0.2 \mathrm{~mm}$ ). The freestanding films with thickness of $5-9 \mathrm{~mm}$ can be easily 
peeled off from the filtration paper after drying about $12 \mathrm{~h}$ in an oven at $50^{\circ} \mathrm{C}$. A series of MTM/PVA/NFX ternary composite films with different MTM/NFX ratios were prepared by altering the volume ratio of NFX suspension to MTM/PVA solution.

\section{RESULTS AND DISCUSSION}

To fabricate the artificial nacre-like MTM/PVA/NFX ternary composite films, NFX is first prepared by a hydrothermal reaction. As shown in Fig. S1 (Supporting information), the products are composed of ultrathin nanobelts. The average diameter is in the range of $20-80 \mathrm{~nm}$, and the length reaches up to tens of micrometers. The energy-dispersive X-ray (EDX) analysis of the synthesized sample indicates the presence of calcium, silicon and oxygen elements (Fig. S2a). All the diffraction peaks in the spectrum can be indexed to the monoclinic phase of calcium silicate hydrate (JCPDS No. 23-0125) (Fig. S2b), indicating the absence of impurities in the asprepared NFX sample. MTM powers dispersed in distilled water were exfoliated into nanosheets with a thickness of about $1 \mathrm{~nm}$ simply by mechanical agitation (Figs S3 and S4a, b). Then, PVA solution and NFX suspension were successively added into the MTM dispersion, followed by vacuum filtration to remove the solvent (Scheme 1a, b). Here, PVA molecules were selected as the mortar to link MTM and NFX because of the strong hydrogen bonds and $\mathrm{Al}-\mathrm{O}-\mathrm{C}$ covalent bonds between PVA and the nanoscale building blocks (Scheme 1c) [17,38]. The polymers on the nanoscale building blocks stabilize the colloid dispersion and reinforce the interphase between MTM and NFX in the composite films (Scheme 1d). In order to confirm the coating of PVA molecules on MTM surface, AFM measurements were performed as exhibited in Fig. S4c, d. The thickness of the MTM nanosheets was increased from $2.2 \mathrm{~nm}$ to $3.5 \mathrm{~nm}$, which can be ascribed to a thin layer of adsorbed PVA [29]. The artificial MTM/ PVA/NFX ternary composite films are visually uniform and smooth, and exhibit high flexibility (Scheme 1e-h), which implies good mechanical properties.

The composition of the as-prepared MTM/PVA/NFX ternary composite film was characterized by Fourier transform infrared spectroscopy (FTIR), thermogravimetric (TG) analysis and wide/small angle XRD patterns. As shown in Fig. S5a, the absorption peak at $3416 \mathrm{~cm}^{-1}$ is attributed to the hydrogen bond, indicating the adsorption of PVA on the MTM and NFX surface. The peak at $846 \mathrm{~cm}^{-1}$ of the MTM/PVA/NFX composite suggests the formation of Al-O-C bonds between MTM and PVA $[17,38]$. The content of PVA molecules adhered on the

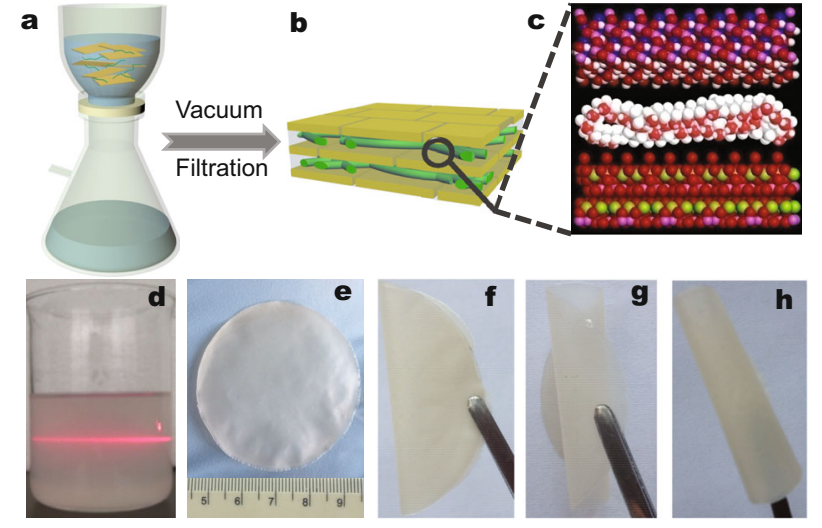

Scheme 1 (a) Fabrication process of the artificial nacre-like MTM/ PVA/NFX ternary composite film by vacuum filtration; (b) layered structure formed via alternative stacking of MTM nanosheets and NFX network layers; (c) structural model proposed for the artificial ternary composite film, in which MTM nanosheets and NFX fibrils are adhered by PVA molecules; (d) digital photo of the well-dispersed MTM/PVA/ NFX mixed aqueous suspension; (e-h) digital photos of the artificial MTM/PVA/NFX ternary composite films.

building blocks was measured by TG analysis (Fig. S5b). The mass percentage of the adsorbed PVA in the typical ternary composite film is about 18.6 wt.\%, which is comparable to the organic content in nacre, and other artificial nacre-like films $[21,23,40]$. From the wide-angle XRD pattern (Fig. S6a), it can be concluded that the building blocks of MTM and NFX are successfully combined in the ternary composite film. The intercalation of NFX into MTM layers is confirmed by small-angle XRD pattern as shown in Fig. S6b, c. The interlayer distance of the artificial MTM/PVA binary composite film is about $0.9 \mathrm{~nm} \quad\left(2 \theta=5.1^{\circ}\right)$. For the ternary MTM/PVA/NFX composite film, the reflection peak is absent in between $1^{\circ}-10^{\circ}$, possibly because the insertion of NFX increases the interlayer distance of MTM layers. The above results confirm that the ternary MTM/PVA/NFX composite film is assembled successfully by stacking $1 \mathrm{D}$ NFX network and 2D MTM nanosheets with the adhesion of PVA molecules.

The microstructure of the artificial MTM/PVA/NFX ternary composite films was investigated by SEM. The composite film presents a layered architecture with strong in-plane orientation of MTM platelets (Fig. 1a, b), similar to the brick-and-mortar structure of nacre. NFX fibrils are located in the space between adjacent MTM platelets. The corresponding element mappings (Fig. 1c-h) suggest the homogeneous distribution of MTM and NFX in the ternary composite films with low polymer filler loading. The EDX spectrum of the cross-sectional ternary com- 

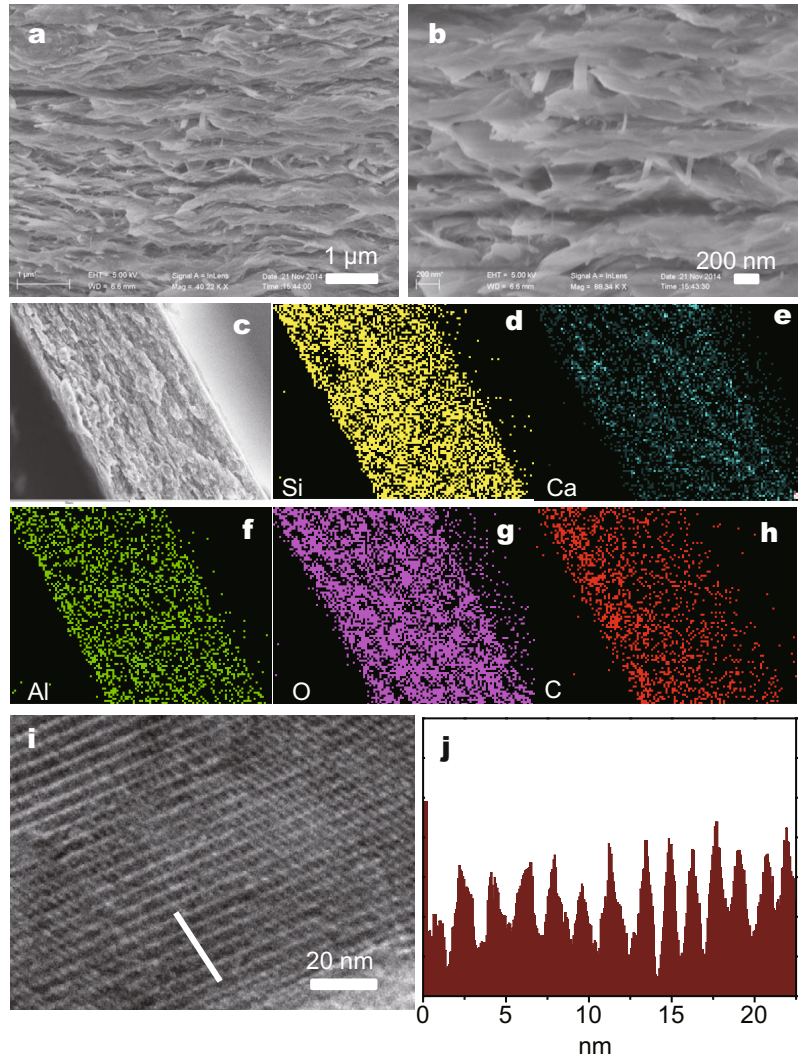

Figure 1 (a, b) Different magnification of cross-sectional SEM images; (c-h) element mapping patterns; (i) cross-sectional HRTEM image of the typical artificial nacre-like MTM/PVA/NFX ternary composite film (the mass ratio of MTM/NFX is 7.5:1), showing the alternating hard and soft layers; ( $\mathrm{j}$ ) the grey scale analysis of the section marked in (i).

posite films illustrates the signals originating from MTM (sodium, magnesium, aluminum, silicon and oxygen), PVA (carbon and oxygen) and NFX (calcium, silicon and oxygen) (Fig. S7). The surface topography of the ternary composite films (Fig. S8) reveals an irregularity surface with minor nanoscale roughness, which implies the uniform dispersion of NFX on MTM platelets. Specifically, high resolution TEM (HRTEM) images of a highly aligned lamella exhibits the alternating hard/soft stacking (Fig. 1i). The grey scale analysis (Fig. 1j) shows the spacing of the layers in the range of $1.7-2.5 \mathrm{~nm}$, which is larger than that of MTM/PVA film $(0.9 \mathrm{~nm})$, and further confirms the intercalation of NFX into MTM layers.

The microstructures of the artificial MTM/PVA/NFX ternary composite films with different mass ratios of NFX/MTM were also investigated by SEM. When the mass ratio of MTM/NFX is 15:1, MTM platelets are densely stacked into a laminated microstructure (Fig. 2a). When the mass ratio of MTM/NFX decreases to 5:1 or $3: 1$, the composite films display a lamellar architecture

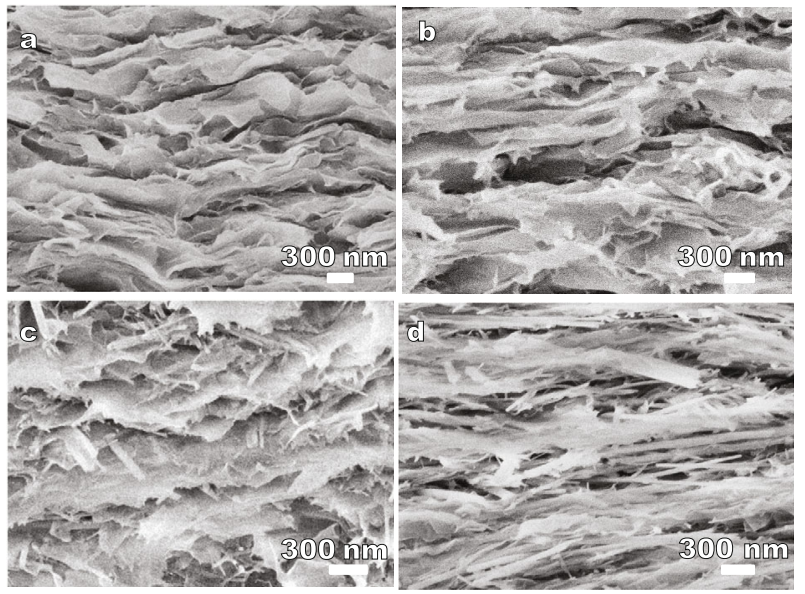

Figure 2 SEM images of the cross-sectional artificial nacre-like MTM/ PVA/NFX ternary composite films with different MTM/NFX mass ratios, i.e., (a) 15:1, (b) 5:1, (c) 3:1 and (d) 1:1, respectively.

even intercalated amounts of rigid NFX (Fig. 2b, c). AFM images (Fig. S9) of the ternary composite film with MTM/ NFX mass ratio of 5:1 further evidence the close contact between NFX and MTM favorable for the fabrication of the lamellar architectures. Further decrease of the mass ratio of MTM/NFX to 1:1 result in larger spacing between adjacent MTM platelets and NFX fibers (Fig. 2c, d). The above results further confirm that the 1D NFX network layer and 2D MTM nanosheet layer are alternatively stacked to form a nacre-like architecture in the obtained ternary composite films.

The mechanical properties of the nacre-like MTM/ PVA/NFX composite films were evaluated by the tensile stress-strain tests (Fig. 3). The tensile strength and toughness of the typical artificial MTM/PVA/NFX ternary composite films are $(241.8 \pm 10.2) \mathrm{MPa}$, and $(5.85$ $\pm 0.46) \mathrm{MJ} \mathrm{m}^{-3}$, respectively (Fig. 3a), both of which are much higher than that of the unitary NFX network film $\left((8.0 \pm 2.3) \mathrm{MPa}\right.$, and $\left.(0.69 \pm 0.12) \mathrm{MJ} \mathrm{m}^{-3}\right)$ and the binary MTM/PVA composite films $((80.6 \pm 8.3) \mathrm{MPa}$, and (5.23 $\pm 0.54) \mathrm{MJ} \mathrm{m}^{-3}$ ) prepared via the vacuum filtration. The prominent mechanical properties of the artificial MTM/ PVA/NFX ternary composite films can be attributed to the synergistic toughening from the hydrogen bonding and $\mathrm{Al}-\mathrm{O}-\mathrm{C}$ covalent bonds among the $1 \mathrm{D}$ and $2 \mathrm{D}$ building blocks. The atomic model (Fig. 3b) reveals that numerous $\mathrm{SiO}_{4}$ tetrahedrons on NFX and MTM surface can interact with the $\mathrm{OH}$ groups in PVA molecules to form hydrogen bonds. In addition, the close adhesion of PVA molecules onto the MTM surface leads to the formation of Al-O-C covalent bonds [17,38]. To investigate the contribution of hydrogen bonding on the toughening 

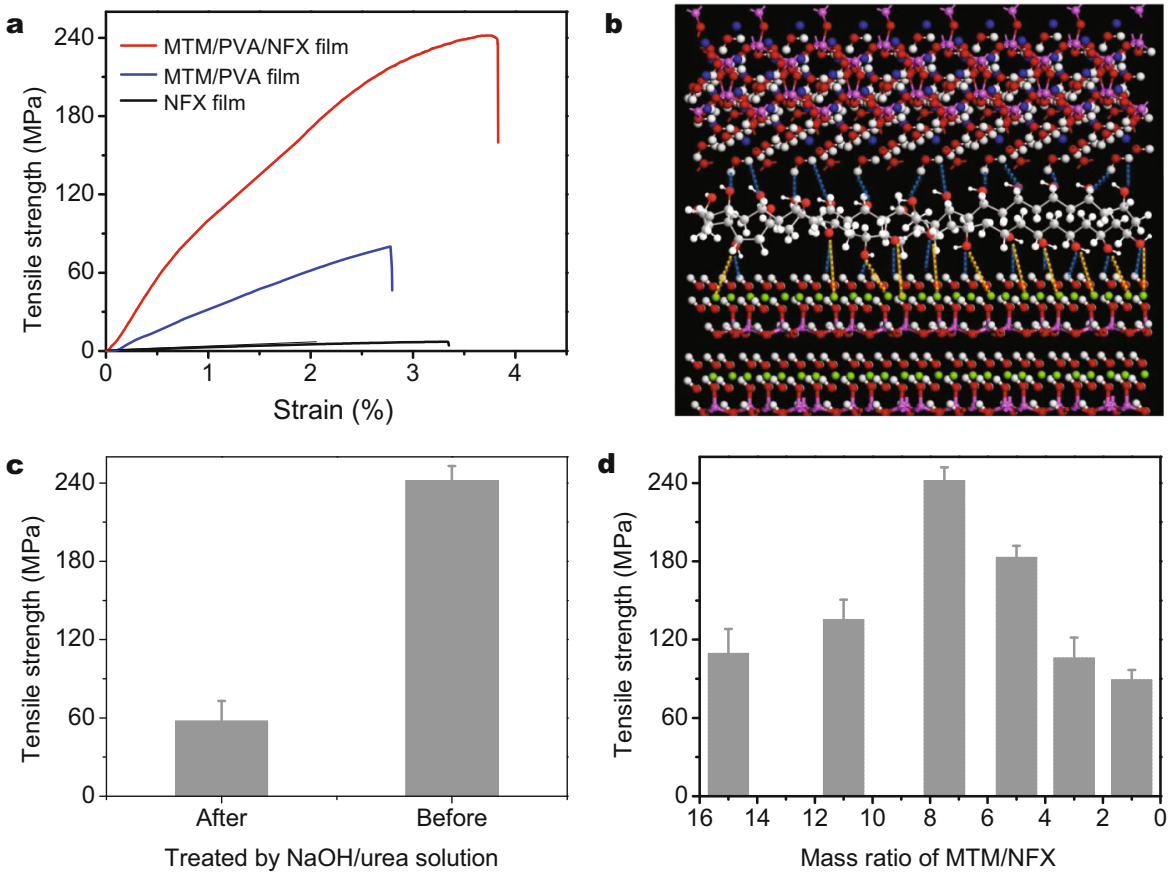

Figure 3 (a) Tensile strength-strain curves of the composite films; (b) atomic model of the composite film showing the adhesion of PVA on MTM and NFX surface through hydrogen bonds and Al-O-C covalent bonds. Al green, Si pink, O red, $\mathrm{C}$ gray, Ca blue, and $\mathrm{H}$ white; (c) comparison of the tensile strength of the composite film before and after treated by $\mathrm{NaOH}$ urea solution; (d) comparison of the tensile strength of the artificial MTM/ PVA/NFX ternary composite films with different mass ratios of MTM and NFX.

effect, the typical artificial MTM/PVA/NFX ternary composite film was treated with alkaline urea aqueous solution. As reported, because the hydrogen bond can be destroyed in the solution [41], the corresponding tensile strength and toughness of the samples treated with alkaline urea solution are $(58.4 \pm 5.2) \mathrm{MPa}$ and $(1.50 \pm 0.21)$ $\mathrm{MJ} \mathrm{m}^{-3}$, respectively (Fig. $3 \mathrm{c}$ and Fig. S10), both of which are lower than that of the untreated sample. The treated MTM/PVA/NFX ternary composite film exhibits a larger interlayer distance, which implies the breakage of hydrogen bonds between PVA and the inorganic building blocks (Fig. S10). The result suggests that hydrogen bonding, compared to the covalent bonds between PVA and MTM, contributes more to the toughening mechanism of the ternary composite films. In order to further illustrate the synergistic toughening effect on the ternary composite films, the mechanical properties of the artificial MTM/PVA/NFX ternary composite films with different NFX/MTM mass ratios were also evaluated (Figs. 3d and S11). The tensile strengths of the artificial MTM/PVA/NFX ternary composite films initially increase with the mass ratio, which are possibly aroused by the synergistic toughening mechanism from their building blocks. After reaching a maximum, the tensile strength gradually decreases with further mass ratio increase (Table S1). The poor mechanical properties of the NFX hinder the tensile strength performance of the composite films with higher NFX content. These results indicate that the synergistic toughening is also relied on their component mass ratio. Consequently, with the synergistic toughening effect, the artificial MTM/PVA/NFX ternary composite films show an outstanding mechanical performance in spite of its high inorganic content.

The artificial MTM/PVA/NFX ternary composite films exhibit reconciled strength and toughness in comparison with previously reported binary clay/polymer composites. The ultimate stress $((241.8 \pm 10.2) \mathrm{MPa})$ of our MTM/ PVA/NFX ternary composite film is approximately 2-3 fold higher than that of dentin $((105 \pm 16.4) \mathrm{MPa})$, and bone $(150 \mathrm{MPa})$. The toughness $\left((5.85 \pm 0.46) \mathrm{MJ} \mathrm{m}^{-3}\right)$ is over 9-fold higher than that of dentin, 15 times higher than that of bone [42]. The tensile strength is 1.2-6.2 times higher than that of the reported binary composite films [18,23,30,43-47]. Meanwhile, the toughness is also superior to most of the previously reported binary films $[18,23,30,39,42-47]$. Although the strength and the toughness are mutually exclusive in many artificial materials, a good balance between these two properties in 


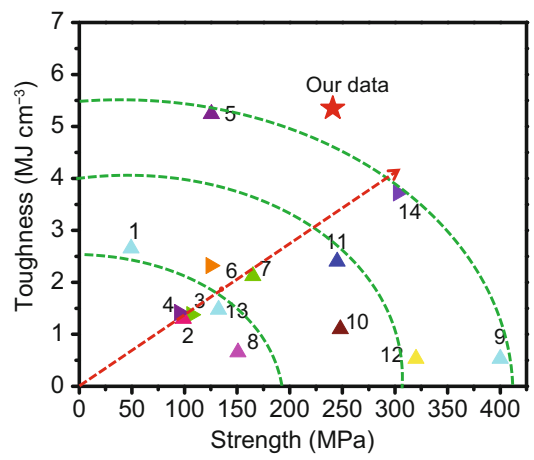

1 MTM/PEO (Sehaqui, 2013, [42])

2 MTM/chitosan (Yao, 2010, [23])

3 MTM/PDDA1 (Walther, 2010, [48])

4 MTM/CMC1 (Liu, 2013, [45])

5 MTM/HEC (Sehaqui, 2013, [42])

6 MTM/NFC1 (Liu, 2011, [43])

7 MTM/PVA1 (Walther, 2010, [29])

8 MTM/PDDA2 (Martikainen, 2013, [44])

9 MTM/PVA2 (Podsiadlo, 2007, [38])

9 MTM/PVA2 (Podsiadlo, 2007, [38])
10 MTM/PVA3 (Walther, 2010, [29])

10 MTM/PVA3 (Walther, 2010, [29])

12 MTM/PVA4 (Podsiadlo, 2008, [46])

$13 \mathrm{MTM} / \mathrm{NFC} /$ chitosan (Liu, 2012, [40])

14 MTM/NFC/PVA (Wang, 2014, [12])

Our data: MMT/PVA/NFX

Figure 4 Comparison of the mechanical properties of the as-prepared artificial MTM/PVA/NFX ternary composite film (mass ratio of MTM to NFX: 7.5:1) and previously reported layered MTM/polymer composites. The arrow presents the overall desired direction for the composite reinforcement. Abbreviations: PEO, polyethylene oxide; PDDA, poly(diallyldimethyl-ammonium chloride); CMC, sodium carboxymethyl cellulose; HEC, hydroxyethyl cellulose; NFC, nanofibrillar cellulose; PVA, poly(vinyl alcohol).
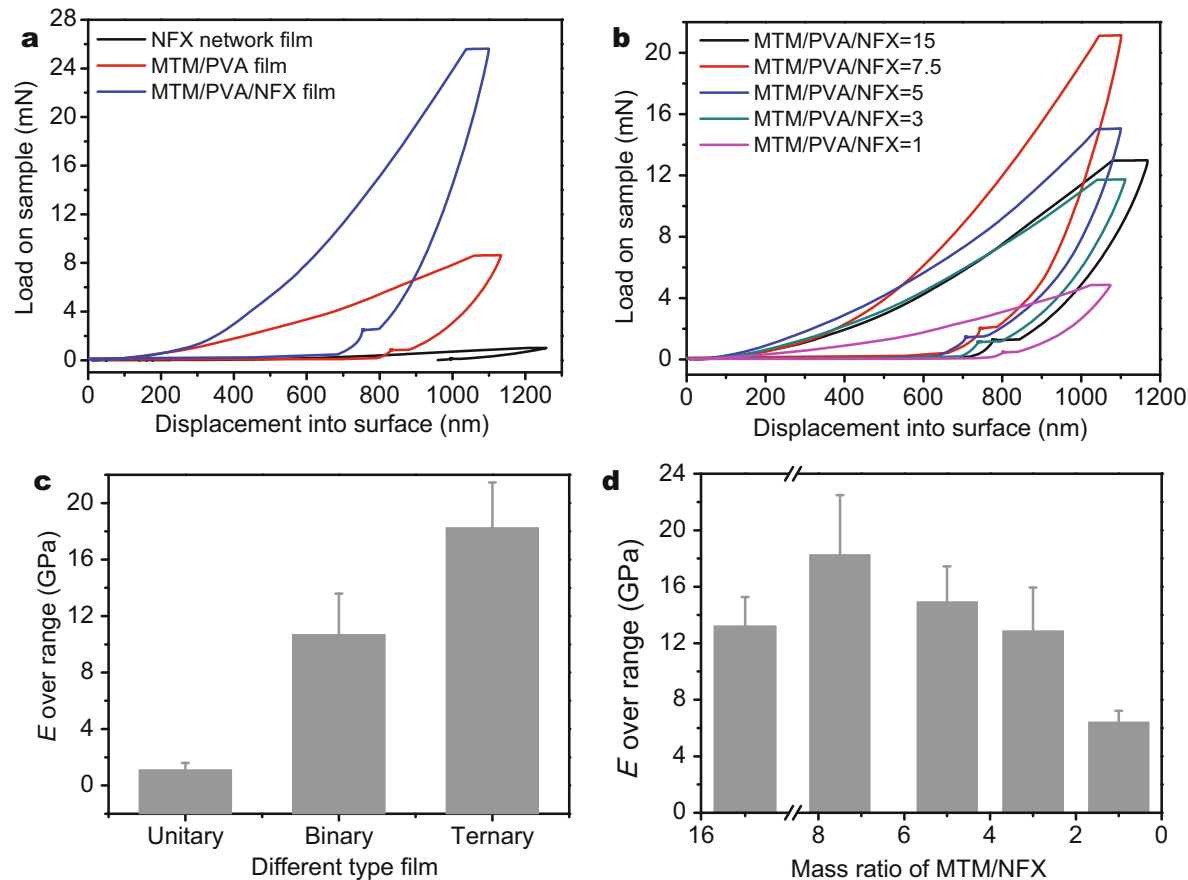

Figure 5 Representative load-displacement curves of different films (a), the MTM/PVA/NFX ternary composite films with different MTM/NFX mass ratios (b). Comparison of the hardness of different films (c), and the MTM/PVA/NFX ternary composite films with different MTM/NFX mass ratios (d).

our ternary films (Fig. 4) can be ascribed to the synergistic toughening mechanism of the ternary structure. The as-prepared MTM/PVA/NFX ternary composite films show an extraordinary mechanical performances in comparison with other ternary composite films (MTM/ $\mathrm{PVA} /$ chistosan, MTM/PVA/cellulose) containing higher content of organic filler (Table S2) [12,39].

We further investigate the mechanical performances of the artificial composite films by nanoindentation tests.
Compared with the unitary NFX network films and the binary MTM/PVA composite films, the typical artificial MTM/PVA/NFX ternary composite films display a prominent mechanical performance with the hardness up to $(18.3 \pm 2.3) \mathrm{GPa}$, which is nearly twice as high as the binary MTM/PVA films ((10.7 \pm 2.1$) \mathrm{GPa})$, and 16 times

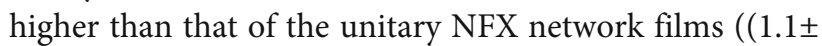
0.5 ) GPa) (Fig. 5a). Such an obvious enhancement can be attributed to the strong interface interactions in the 
ternary composite films. As the mass ratios of NFX/MTM in the ternary composite films vary, their load-displacement curves also change (Fig. 5b). Their hardness increases from $(13.2 \pm 2.1) \mathrm{GPa}$ to $(18.3 \pm 2.3) \mathrm{GPa}$ with the mass ratio of MTM/NFX decrease from 15:1 to 7.5:1. With further increase of the NFX content in the composite films, their hardness begins to decrease gradually due to the brittleness of the inorganic NFX $[16,20]$. The hardness of the different composite films is summarized in Fig. $5 c$, d. The results suggest that the synergistic effect of the building blocks promotes the enhancement of the hardness in spite of the brittleness of the NFX.

The optical properties of the artificial composite films were also investigated (Fig. S12a). The typical artificial MTM/PVA/NFX ternary composite films present as high as $45 \%-65 \%$ light transmittance relative to the binary MTM/PVA composite films (40\%-55\%) and the unitary NFX network films (lower than 5\%) in visible light range with similar thickness. The layered architecture with strong in-plane orientation of the artificial MTM/PVA/ NFX ternary composite films leads to higher light transmittance than the randomly dispersed unitary NFX network films [23]. When increasing the NFX contents in the ternary composite films, their light transmittance gradually decreases (Fig. S12b) because higher NFX content increases the light scattering between the MTM interlayers. The photographs of the artificial MTM/PVA/ NFX ternary composite films with different NFX/MTM mass ratios also show that the ternary composite films gradually become less transparent with increasing the NFX content (Fig. S12c).

\section{CONCLUSIONS}

In summary, artificial MTM/PVA/NFX ternary composite films with nacre-like architecture, which are composed of a NFX network and MTM nanosheets, can be fabricated by a facile vacuum filtration assembly. Compared to conventional binary MTM/PVA composite films, the ternary composite films exhibit lower organic filler content (comparable to natural nacre), well-aligned lamellar architecture, integration of high tensile strength and toughness, and adjustable optical transmittance. This method provides a new concept for designing high performance composite films based on the synergistic mechanism of various nanoscale building blocks.

Received 9 June 2017; accepted 21 August 2017; published online 28 September 2017

1 Ritchie RO. The conflicts between strength and toughness. Nat Mater, 2011, 10: 817-822
2 Mao LB, Gao HL, Yao HB, et al. Synthetic nacre by predesigned matrix-directed mineralization. Science, 2016, 354: 107-110

3 Zhao HW, Guo L. Synthesis and applications of layered structural composites inspired by nacre. Chin Sci Bull, 2016, 62: 576-589

4 Grossman M, Bouville F, Erni F, et al. Mineral nano-interconnectivity stiffens and toughens nacre-like composite materials. Adv Mater, 2017, 29: 1605039

5 Cheng Q, Jiang L, Tang Z. Bioinspired layered materials with superior mechanical performance. Acc Chem Res, 2014, 47: 12561266

6 Espinosa HD, Rim JE, Barthelat F, et al. Merger of structure and material in nacre and bone-Perspectives on de novo biomimetic materials. Prog Mater Sci, 2009, 54: 1059-1100

7 Barthelat F. Nacre from mollusk shells: a model for high-performance structural materials. Bioinspir Biomim, 2010, 5: 035001

8 Das P, Malho JM, Rahimi K, et al. Nacre-mimetics with synthetic nanoclays up to ultrahigh aspect ratios. Nat Commun, 2015, 6: 5967

9 Song F, Soh AK, Bai YL. Structural and mechanical properties of the organic matrix layers of nacre. Biomaterials, 2003, 24: 36233631

10 Bouville F, Maire E, Meille S, et al. Strong, tough and stiff bioinspired ceramics from brittle constituents. Nat Mater, 2014, 13: 508-514

11 Meyers MA, McKittrick J, Chen PY. Structural biological materials: critical mechanics-materials connections. Science, 2013, 339: 773779

12 Wang J, Cheng Q, Lin L, et al. Synergistic toughening of bioinspired poly(vinyl alcohol)-clay-nanofibrillar cellulose artificial nacre. ACS Nano, 2014, 8: 2739-2745

13 Laaksonen P, Walther A, Malho JM, et al. Genetic engineering of biomimetic nanocomposites: diblock proteins, graphene, and nanofibrillated cellulose. Angew Chem Int Ed, 2011, 50: 8688-8691

14 Suzuki M, Saruwatari K, Kogure T, et al. An acidic matrix protein, pif, is a key macromolecule for nacre formation. Science, 2009, 325: 1388-1390

15 Cheng Q, Jiang L. Science behind nacre: matrix-directed mineralization at ambient condition. Sci China Mater, 2016, 59: 889-891

16 Yan YX, Yao HB, Smart SE, et al. Ultrathin hybrid films of polyoxohydroxy clusters and proteins: layer-by-layer assembly and their optical and mechanical properties. Langmuir, 2014, 30: 52485255

17 Tang Z, Kotov NA, Magonov S, et al. Nanostructured artificial nacre. Nat Mater, 2003, 2: 413-418

18 Yao HB, Fang HY, Tan ZH, et al. Biologically inspired, strong, transparent, and functional layered organic-inorganic hybrid films. Angew Chem Int Ed, 2010, 49: 2140-2145

19 Shu Y, Yin P, Liang B, et al. Artificial nacre-like gold nanoparticles -layered double hydroxide-poly(vinyl alcohol) hybrid film with multifunctional properties. Ind Eng Chem Res, 2015, 54: 89408946

20 Chen K, Shi B, Yue Y, et al. Binary synergy strengthening and toughening of bio-inspired nacre-like graphene oxide/sodium alginate composite paper. ACS Nano, 2015, 9: 8165-8175

21 Cheng Q, Wu M, Li M, et al. Inside back cover: ultratough artificial nacre based on conjugated cross-linked graphene oxide. Angew Chem Int Ed, 2013, 52: 3775-3775

22 Wang J, Lin L, Cheng Q, et al. A strong bio-inspired layered PNIPAM-clay nanocomposite hydrogel. Angew Chem, 2012, 124: 4754-4758 
23 Yao HB, Tan ZH, Fang HY, et al. Artificial nacre-like bionanocomposite films from the self-assembly of chitosan-montmorillonite hybrid building blocks. Angew Chem Int Ed, 2010, 49: 10127-10131

24 Bonderer LJ, Studart AR, Gauckler LJ. Bioinspired design and assembly of platelet reinforced polymer films. Science, 2008, 319: 1069-1073

25 Lin TH, Huang WH, Jun IK, et al. Bioinspired assembly of colloidal nanoplatelets by electric field. Chem Mater, 2009, 21: 20392044

26 Munch E, Launey ME, Alsem DH, et al. Tough, bio-inspired hybrid materials. Science, 2008, 322: 1516-1520

27 Wang J, Cheng Q, Lin L, et al. Understanding the relationship of performance with nanofiller content in the biomimetic layered nanocomposites. Nanoscale, 2013, 5: 6356-6362

28 Liang $\mathrm{B}$, Zhao $\mathrm{H}$, Zhang Q, et al. $\mathrm{Ca}^{2+}$ enhanced nacre-inspired montmorillonite-alginate film with superior mechanical, transparent, fire retardancy, and shape memory properties. ACS Appl Mater Interfaces, 2016, 8: 28816-28823

29 Walther A, Bjurhager I, Malho JM, et al. Large-area, lightweight and thick biomimetic composites with superior material properties via fast, economic, and green pathways. Nano Lett, 2010, 10: $2742-$ 2748

30 Zhong D, Yang Q, Guo L, et al. Fusion of nacre, mussel, and lotus leaf: bio-inspired graphene composite paper with multifunctional integration. Nanoscale, 2013, 5: 5758-5764

31 Nudelman F, Chen HH, Goldberg HA, et al. Spiers Memorial Lecture: Lessons from biomineralization: comparing the growth strategies of mollusc shell prismatic and nacreous layers in Atrina rigida. Faraday Discuss, 2007, 136: 9

32 Levi-Kalisman Y, Falini G, Addadi L, et al. Structure of the nacreous organic matrix of a bivalve mollusk shell examined in the hydrated state using cryo-TEM. J Struct Biol, 2001, 135: 8-17

33 Weiner S, Traub W, Parker SB. Macromolecules in mollusc shells and their functions in biomineralization [and discussion]. Philos Trans R Soc B-Biol Sci, 1984, 304: 425-434

34 Cheng Q, Duan J, Zhang Q, et al. Learning from nature: constructing integrated graphene-based artificial nacre. ACS Nano, 2015, 9: 2231-2234

35 Wan S, Li Y, Peng J, et al. Synergistic toughening of graphene oxide-molybdenum disulfide-thermoplastic polyurethane ternary artificial nacre. ACS Nano, 2015, 9: 708-714

36 Knöller A, Lampa CP, Cube F, et al. Strengthening of ceramicbased artificial nacre via synergistic interactions of $1 \mathrm{D}$ vanadium pentoxide and 2D graphene oxide building blocks. Sci Rep, 2017, 7: 40999

37 Liu M, Jiang L. Dialectics of nature in materials science: binary cooperative complementary materials. Sci China Mater, 2016, 59: 239-246

38 Podsiadlo P, Kaushik AK, Arruda EM, et al. Ultrastrong and stiff layered polymer nanocomposites. Science, 2007, 318: 80-83

39 Lin K, Liu X, Chang J, et al. Facile synthesis of hydroxyapatite nanoparticles, nanowires and hollow nano-structured microspheres using similar structured hard-precursors. Nanoscale, 2011, 3: $3052-3055$
40 Liu A, Berglund LA. Clay nanopaper composites of nacre-like structure based on montmorrilonite and cellulose nanofibersImprovements due to chitosan addition. Carbohyd Polym, 2012, 87: $53-60$

41 Cai J, Zhang L, Liu S, et al. Dynamic self-assembly induced rapid dissolution of cellulose at low temperatures. Macromolecules, 2008, 41: 9345-9351

42 Sehaqui H, Kochumalayil J, Liu A, et al. Multifunctional nanoclay hybrids of high toughness, thermal, and barrier performances. ACS Appl Mater Interfaces, 2013, 5: 7613-7620

43 Liu A, Walther A, Ikkala O, et al. Clay nanopaper with tough cellulose nanofiber matrix for fire retardancy and gas barrier functions. Biomacromolecules, 2011, 12: 633-641

44 Martikainen L, Walther A, Seitsonen J, et al. Deoxyguanosine phosphate mediated sacrificial bonds promote synergistic mechanical properties in nacre-mimetic nanocomposites. Biomacromolecules, 2013, 14: 2531-2535

45 Liu A, Berglund LA. Fire-retardant and ductile clay nanopaper biocomposites based on montmorrilonite in matrix of cellulose nanofibers and carboxymethyl cellulose. Eur Polymer J, 2013, 49: 940-949

46 Podsiadlo P, Kaushik AK, Shim BS, et al. Can nature's design be improved upon? High strength, transparent nacre-like nanocomposites with double network of sacrificial cross links. J Phys Chem B, 2008, 112: 14359-14363

47 Zeng X, Ye L, Yu S, et al. Artificial nacre-like papers based on noncovalent functionalized boron nitride nanosheets with excellent mechanical and thermally conductive properties. $\mathrm{Na}$ noscale, 2015, 7: 6774-6781

48 Walther A, Bjurhager I, Malho JM, et al. Supramolecular control of stiffness and strength in lightweight high-performance nacre-mimetic paper with fire-shielding properties. Angew Chim Int Ed, 2010, 49: 6448-6453

49 Das P, Walther A. Ionic supramolecular bonds preserve mechanical properties and enable synergetic performance at high humidity in water-borne, self-assembled nacre-mimetics. Nanoscale, 2013, 5: 9348-9356

Acknowledgements This work was supported by the National Natural Science Foundation of China (51732011 and 21431006), the Foundation for Innovative Research Groups of the National Natural Science Foundation of China (21521001), the National Basic Research Program of China (2014CB931800), and the Users with Excellence and Scientific Research Grant of Hefei Science Center of Chinese Academy of Sciences (2015HSC-UE007 and 2015SRG-HSC038).

Author contributions Li S and Mao L performed the experiments; Li $\mathrm{S}$, Mao L and Yu S wrote the paper. Yu S supervised the projects. All authors contributed to the general discussion.

Conflict of interest The authors declare that they have no conflict of interest.

Supplementary information Supporting information is available in the online version of the paper. 


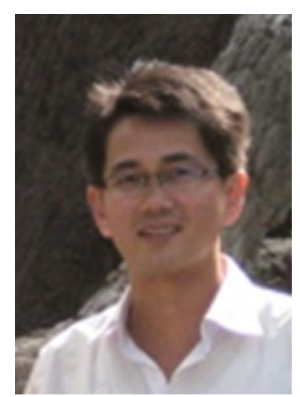

Shi-Kuo Li received his master degree majored in applied chemistry from Anhui University in 2007. Then he completed his $\mathrm{PhD}$ degree at the University of Science and Technology of China under the supervision of Prof. Shu-Hong Yu. His research interest is the bio-inspired fabrication of multilayered composite materials and their performances.

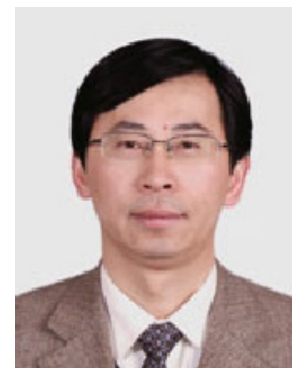

Shu-Hong Yu received his BSc at Hefei University of Technology and his PhD in inorganic chemistry from the University of Science and Technology of China (USTC). He was a postdoctoral fellow with M. Yoshimura (Tokyo Institute of Technology) and a Humboldt Fellow with M. Antonietti and H. Cölfen (MPI of Colloids and Interfaces, Germany). In 2002, he was appointed the Cheung Kong Professor at USTC. Currently, he leads the Division of Nanomaterials \& Chemistry at the Hefei National Laboratory for Physical Sciences at Microscale, USTC. His current research interests include bio-inspired synthesis and self-assembly of new nanostructured materials and nanocomposites, and their related properties. He serves as a senior editor for Langmuir, and an editorial advisory board member of journals Accounts of Chemical Research, Chemistry of Materials, Chemical Science, Materials Horizons, Nano Research, ChemNanoMat, CrystEngComm, and Part. Part. Syst. Charact. His recent awards include Chem. Soc. Rev. Emerging Investigator Award (2010) and Roy-Somiya Medal of the International Solvothermal and Hydrothermal Association (ISHA) (2010).

\section{受生物启发的三元柔性粘土片/聚乙烯醇/硅酸钲纳米纤维复合薄膜的组装及协同增强力学性能研究}

李士阔, 茅柇波, 高怀岭, 姚宏斌, 俞书宏 ${ }^{*}$

摘要 尽管人们在实验室模拟贝壳的多级层状结构方面已经取得了显著进步, 但在理解生物材料独特的强度和韧性的平衡方面仍然有很 大的挑战. 本文利用不同维度的组装单元制备了具有高强度和韧性的三元仿贝壳层状多级结构的粘土片/聚乙烯醇/硅酸锎纳米纤维 (MTM/PVA/NFX)复合薄膜. 这种层状的人工复合薄膜是通过一维的NFX网状层和二维的MTM纳米片层交替堆积而成, 制得的MTM/ PVA/NFX复合薄膜不但强度高 $(241.8 \pm 10.2 \mathrm{MPa})$, 而且韧性好 $\left(5.85 \pm 0.46 \mathrm{MJ} \mathrm{m}{ }^{-3}\right)$. 其数值比一元的NFX网状薄膜和二元的MTM/PVA 复合薄膜高出数倍, 并且优于传统的二元MTM/聚合物复合薄膜. 这可能是由于所制备的复合薄膜中MTM纳米片与NFX纳米纤维产生的 协同增强效应所导致的. 这种基于组分间协同增强的组装方案为设计和构建新型仿贝壳层状多级结构的复合薄膜提供了一种简单的方法. 这种柔性复合膜可望应用于功能涂层、组织工程和膜分离等领域. 